\title{
Just the facts: updates in COVID-19 therapeutics
}

\author{
Brit Long ${ }^{1}\left[\right.$. Stephen Y. Liang ${ }^{2} \cdot$ Hans Rosenberg ${ }^{3} \cdot$ Christopher Hicks $^{4} \cdot$ Michael Gottlieb $^{5}$ \\ Received: 2 December 2020 / Accepted: 24 December 2020 / Published online: 8 February 2021 \\ (c) Canadian Association of Emergency Physicians (CAEP)/ Association Canadienne de Médecine d'Urgence (ACMU) 2021
}

Keywords COVID-19 - Therapeutics · Medications

\section{When should steroids be administered?}

Evidence suggests that glucocorticoids can reduce mortality in patients with COVID-19 who require oxygen supplementation. A randomized controlled trial (RCT) including over 6400 patients found reduced 28-day mortality in patients who received oral or intravenous dexamethasone $6 \mathrm{mg}$ compared with placebo (22.9 vs. $25.7 \%$ ) [1]. A meta-analysis incorporating this study with 6 others found a $32.7 \%$ mortality rate in those receiving glucocorticoids, compared to $41.5 \%$ among the control group [2]. A separate meta-analysis suggests glucocorticoids reduce mortality by $3.1 \%$ and need for mechanical ventilation by $2.8 \%$ [3]. Dexamethasone currently has the most robust evidence as a therapy in COVID-19, and as such, we recommend using dexamethasone $6 \mathrm{mg}$ oral (PO) or intravenous (IV) for patients with COVID-19 requiring oxygen supplementation.

This paper is a follow up and supplement to the previously
published article Long B, Liang SY, Rosenberg H, Hicks C,
Gottlieb M. Just the facts: What drugs are safe and effective
for COVID-19? CJEM. 2020 Sep;22(5):591-594. https://
doi.org/10.1017/CEM.2020.403. PMID: 32438948; PMCID:
PMC7267102. https://doi.org/10.1017/cem.2020.403.

Brit Long

brit.long@yahoo.com

Stephen Y. Liang

syliang@wustl.edu

Hans Rosenberg

hrosenberg@toh.ca

Christopher Hicks

Christopher.Hicks@unityhealth.to

Michael Gottlieb

MichaelGottliebMD@Gmail.com

\section{Is there a benefit to convalescent plasma?}

Convalescent plasma demonstrated promise early in the pandemic [4]. A Cochrane review with 19 studies (2 RCTs) found no clear evidence that convalescent plasma reduces mortality or improves clinical symptoms, with unclear side effects [5]. Thus, it remains uncertain whether convalescent plasma provides any benefit in patients with COVID-19, and we do not recommend it for routine use at this time [4].

\section{Is there a benefit to remdesivir?}

Remdesivir is currently approved by the United State Food and Drug Administration (U.S. FDA) for use in COVID-19 [4]. One of the largest studies to date is the ACTT-1 trial, which included 1062 patients with confirmed COVID-19, of which $85 \%$ were classified as severe [6]. Patients receiving remdesivir demonstrated faster time to recovery (10 vs 15 days), but on subgroup analysis, this was only present in patients on low flow oxygen. This specific subgroup also demonstrated reduced mortality. Among those who were critically ill on mechanical ventilation or extracorporeal membrane oxygenation, there was no difference in time to recovery or mortality. In patients with non-severe disease,

1 Present Address: Department of Emergency Medicine, Brooke Army Medical Center, 3841 Roger Brooke Dr, Fort Sam Houston, TX 78234, USA

2 Divisions of Emergency Medicine and Infectious Diseases, Washington University School of Medicine, 660 S. Euclid Ave, St. Louis, MO 63110, USA

3 Department of Emergency Medicine, University of Ottawa, Ottawa, ON, Canada

4 Division of Emergency Medicine, Department of Medicine, University of Toronto, Toronto, ON, Canada

5 Department of Emergency Medicine, Rush University Medical Center, Chicago, IL 60612, USA 
there is also no clear benefit. The ACTT-1 trial included 119 patients with mild-moderate disease, with no difference in time to recovery with remdesivir [6]. In a separate RCT of 584 patients with moderate COVID-19, the authors found no significant difference in clinical status based on a 7-point ordinal scale after a 10-day course of remdesivir [7]. Another RCT found no difference in mortality [8]. Remdesivir has only shown benefit in a very narrow clinical spectrum, and therefore routine use for all patients is not recommended.

\section{What is bamlanivimab?}

Bamlanivimab is a monoclonal antibody which recently received an emergency use authorization by the U.S. FDA for treatment of patients with confirmed mild to moderate COVID-19 within 10 days of symptom onset and risk of progressing to severe disease [9]. It requires intravenous infusion and is not authorized for patients requiring supplemental oxygen or those who are hospitalized [9]. As of late November 2020, the evidence is controversial in its ability to reduce the viral load or reduce hospitalizations, and the Infectious Diseases Society of America recommends against its routine use $[10,11]$. Other monoclonal antibodies, alone or in combination, are under evaluation.

\section{What does the evidence suggest for antimalarials?}

While initially purported as a treatment strategy early in the pandemic, antimalarial agents (e.g., hydroxychloroquine, chloroquine) have not demonstrated significant benefit, while demonstrating significant risk of harm, including severe cardiac arrhythmias [3]. As a result, antimalarial agents are no longer recommended for the treatment of COVID-19.

\section{Key points}

1. For patients with COVID-19 requiring supplemental oxygen, glucocorticoids (dexamethasone $6 \mathrm{mg}$ ) are recommended.

2. It is uncertain whether convalescent plasma provides any benefit in patients with COVID-19.

3. Remdesivir may benefit patients on low flow oxygen, but benefit is not present in other populations. Routine use for all patients is not recommended.
4. It is uncertain whether bamlanivimab or other monoclonal antibodies reduce viral load or need for hospitalization.

5. Hydroxychloroquine and chloroquine have no benefit and may result in harm; thus, they are not recommended.

Infographic. COVID-19 therapeutics updates.

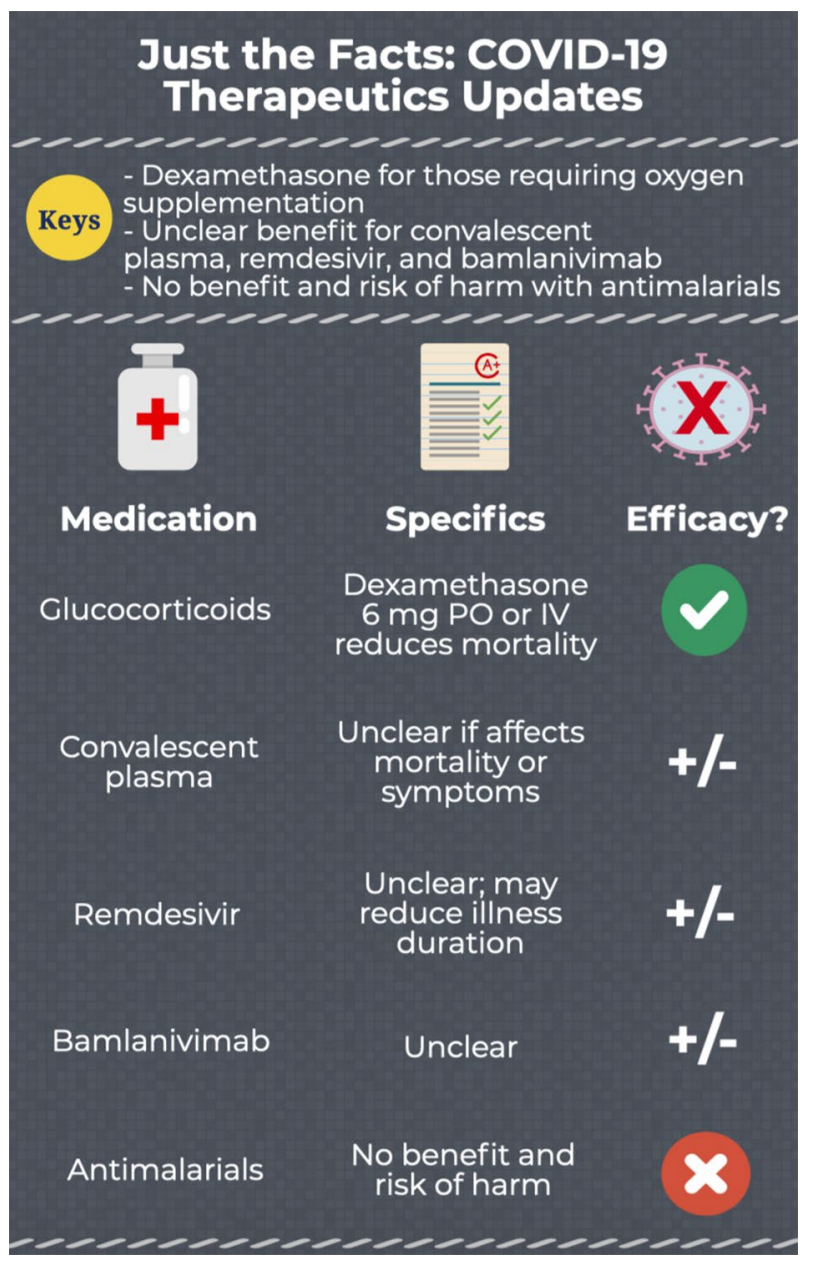

Acknowledgements $\mathrm{BL}, \mathrm{SL}, \mathrm{CH}, \mathrm{HR}$, and $\mathrm{MG}$ conceived the idea for this manuscript, obtained permission for submission from Dr. Paul Atkinson, and contributed substantially to the writing and editing of the review. This manuscript did not utilize any grants or funding, and it has not been presented in abstract form. This clinical review has not been published, it is not under consideration for publication elsewhere, its publication is approved by all authors and tacitly or explicitly by the responsible authorities where the work was carried out, and that, if accepted, it will not be published elsewhere in the same form, in English or in any other language, including electronically without the written consent of the copyright holder. This review does not reflect the views or opinions of the U.S. Government, Department of Defense, U.S. Army, U.S. Air Force, or SAUSHEC EM Residency Program. 


\section{Compliance with ethical standards}

Conflict of interest None.

\section{References}

1. RECOVERY Collaborative Group, Horby P, Lim WS, Emberson JR, et al. Dexamethasone in hospitalized patients with Covid-19preliminary report. N Engl J Med. 2020. https://doi.org/10.1056/ NEJMoa2021436.

2. WHO Rapid Evidence Appraisal for COVID-19 Therapies (REACT) Working Group, Sterne JAC, Murthy S, Diaz JV, et al. Association between administration of systemic corticosteroids and mortality among critically ill patients with COVID19: a meta-analysis. JAMA. 2020;324(13):1-13. https://doi. org/10.1001/jama.2020.17023.

3. Siemieniuk RA, Bartoszko JJ, Ge L, et al. Drug treatments for covid-19: living systematic review and network meta-analysis. BMJ. 2020;30(370):m2980. https://doi.org/10.1136/bmj.m2980.

4. Long B, Liang SY, Rosenberg H, Hicks C, Gottlieb M. Just the facts: what drugs are safe and effective for COVID-19? CJEM. 2020;22(5):591-4. https://doi.org/10.1017/cem.2020.403.

5. Piechotta V, Chai KL, Valk SJ, et al. Convalescent plasma or hyperimmune immunoglobulin for people with COVID19: a living systematic review. Cochrane Database Syst Rev.
2020;7(7):CD013600. https://doi.org/10.1002/14651858.CD013 600.pub2.

6. Beigel JH, Tomashek KM, Dodd LE, ACTT-1 Study Group Members, et al. Remdesivir for the treatment of Covid-19-final report. N Engl J Med. 2020. https://doi.org/10.1056/NEJMoa2007764 (Epub ahead of print. PMID: 32445440).

7. Spinner CD, Gottlieb RL, Criner GJ, GS-US-540-5774 Investigators, et al. Effect of remdesivir vs standard care on clinical status at 11 days in patients with moderate COVID-19: a randomized clinical trial. JAMA. 2020;324(11):1048-57. https://doi. org/10.1001/jama.2020.16349 (PMID: 32821939).

8. WHO Solidarity trial consortium. Repurposed antiviral drugs for COVID-19-interim WHO SOLIDARITY trial results. Available at https://www.medrxiv.org/content/10.1101/2020.10.15.20209 817v1.full.pdf. Accessed 17 Oct 2020.

9. Fact Sheet for Health Care Providers Emergency Use Authorization (EUA) of Bamlanivimab. FDA. Available at https://www.fda. gov/media/143603/download. Accessed 30 Nov 2020.

10. Chen P, Nirula A, Heller B, Gottlieb RL, Boscia J, Morris J, BLAZE-1 Investigators, et al. SARS-CoV-2 neutralizing antibody LY-CoV555 in outpatients with Covid-19. N Engl J Med. 2020. https://doi.org/10.1056/NEJMoa2029849.

11. Bhimraj A, Morgan RL, Shumaker AH, Lavergne V, Baden L, Cheng VC, et al. Infectious Diseases Society of america guidelines on the treatment and management of patients with COVID19. Clin Infect Dis. 2020. https://doi.org/10.1093/cid/ciaa478. 